$1-19-2021$

\title{
Comprehensive Echocardiographic Findings in Critically III COVID-19 Patients With or Without Prior Cardiac Disease
}

\author{
Renuka Jain \\ Pedro D. Salinas \\ Stacie Kroboth \\ Abigail Kaminski \\ Sarah Roemer \\ Ana Cristina Perez Moreno \\ Bijoy K Khandheria
}

Follow this and additional works at: https://aah.org/jpcrr

Part of the Cardiology Commons, Cardiovascular Diseases Commons, Cardiovascular System Commons, Clinical Epidemiology Commons, Critical Care Commons, Diagnosis Commons, Health Services Research Commons, and the Infectious Disease Commons

\section{Recommended Citation}

Jain R, Salinas PD, Kroboth S, Kaminski A, Roemer S, Perez Moreno AC, Khandheria BK. Comprehensive echocardiographic findings in critically ill COVID-19 patients with or without prior cardiac disease. J Patient Cent Res Rev. 2021;8.68-76. doi: 10.17294/2330-0698.1791

Published quarterly by Midwest-based health system Advocate Aurora Health and indexed in PubMed Central, the Journal of Patient-Centered Research and Reviews (JPCRR) is an open access, peer-reviewed medical journal focused on disseminating scholarly works devoted to improving patient-centered care practices, health outcomes, and the patient experience. 


\title{
Comprehensive Echocardiographic Findings in Critically III COVID-19 Patients With or Without Prior Cardiac Disease
}

\author{
Renuka Jain, MD, ${ }^{1}$ Pedro D. Salinas, MD, ${ }^{2}$ Stacie Kroboth, BS, ${ }^{3}$ Abigail Kaminski, BS, RDCS, RVT, \\ Sarah Roemer, BS, RDCS, ${ }^{1}$ Ana Cristina Perez Moreno, MD, PhD, ${ }^{3}$ Bijoy K. Khandheria, MD ${ }^{1}$ \\ ${ }^{1}$ Aurora Cardiovascular and Thoracic Services, Aurora Sinai/Aurora St. Luke's Medical Centers, University of \\ Wisconsin School of Medicine and Public Health, Milwaukee, WI; ${ }^{2}$ Critical Care Services, Aurora Sinai/Aurora St. \\ Luke's Medical Centers, University of Wisconsin School of Medicine and Public Health, Milwaukee, WI; ${ }^{3}$ Advocate \\ Aurora Research Institute, Advocate Aurora Health, Milwaukee, WI
}

\begin{abstract}
Purpose
Coronavirus disease 2019 (COVID-19) presents with a spectrum of disease severity, the most serious cases requiring intensive care. Echocardiography is a front-line tool in evaluating cardiovascular complications of COVID-19 in the intensive care unit (ICU); we analyzed transthoracic echocardiograms obtained from this patient population with state-of-the-art ultrasound technology.
\end{abstract}

Methods

All patients with COVID-19 requiring ICU admission on whom a transthoracic echocardiogram was obtained were included in the study. Focused transthoracic protocols were performed by experienced sonographers. Echocardiographic variables, including speckle-tracking echocardiography, were collected and analyzed. Clinical information was obtained from the electronic medical record. Patients were followed until discharge.

Results

Of 52 total patients (mean age: $59.9 \pm 11.6$ years), 59.6\% were male and $15(29 \%)$ had known prior cardiac disease. Cardiac complications identified on echocardiography were prevalent, occurring in $55.7 \%$ of patients. Patients with known prior cardiac disease were more likely to have new or worsening left ventricular dysfunction. Right ventricular dysfunction was the most common abnormality (assessed qualitatively in 18 cases and with advanced echocardiographic methods in 34 cases). Known prior cardiac disease, right ventricular enlargement, and pulmonary hypertension were significantly associated with morbidity and mortality.

Conclusions Patients requiring intensive care for COVID-19 face significant morbidity and mortality, and cardiac complications occur in the majority of patients admitted to the ICU with COVID-19. Those with known prior cardiac disease fare worse, and other echocardiographic findings (right ventricular enlargement, pulmonary hypertension) are also associated with worse outcomes. State-of-the-art echocardiography performed by experienced sonographers can be critical to identifying cardiac complications and guiding ICU strategy. (J Patient Cent Res Rev. 2021;8:68-76.)

Keywords coronavirus; COVID-19; echocardiography; left ventricle; right ventricle; intensive care unit

Corresponding author: Renuka Jain, MD,

Aurora Cardiovascular and Thoracic Services, 2801 W. Kinnickinnic River Parkway, \#880, Milwaukee, WI 53215 (publishing159@aurora.org) case reports since this virus first presented in human hosts in late 2019. ${ }^{1-5}$ Different clinical presentations, including elevated troponin, myocarditis, myopericarditis, and acute coronary syndrome, have been seen in patients without known prior cardiac disease. Preexisting cardiac disease has been demonstrated to be a significant risk factor for morbidity and mortality in patients who contract COVID-19. ${ }^{6}$ Recent literature also has shown the importance of right ventricular (RV) function in predicting morbidity and mortality in hospitalized patients with COVID-19.,

Echocardiography is a front-line tool for evaluation of cardiovascular complications of COVID-19. It is especially valuable in patients admitted to the ICU, as 
echocardiography can be performed with ease at the bedside. Yet, most published studies used point-of-care ultrasound to evaluate patients with COVID-19 due to concerns about staff safety and machine contamination. There is no study to date that has comprehensively evaluated echocardiographic findings specifically of patients admitted to the ICU with COVID-19, stratified by known prior cardiac disease.

We sought to describe the echocardiographic characteristics of patients presenting with COVID-19 who underwent transthoracic echocardiography (TTE) during their ICU stay and to evaluate potential echocardiographic predictors of morbidity and mortality.

\section{METHODS}

We included all patients admitted to any of our medical center's designated "COVID ICUs" from March to May 2020. Patients were diagnosed with COVID-19 (via positive SARS-CoV-2 polymerase chain reaction test from nasopharyngeal swab) and had at least 1 TTE performed during hospitalization. All included patients had a discharge status (alive and discharged home, dead, or discharged to a long-term care facility). The study was approved by the local institutional review board, and informed consent was waived.

The protocol for TTE was optimized to prioritize the most advanced echocardiographic imaging while ensuring the highest level of protection for the cardiac sonographer. All TTEs were performed at the bedside using the GE Vivid E95 system (GE Healthcare), which was stored in the designated COVID ICU. Keyboard and ultrasound transducer covers were used (Figure 1A). A dedicated group of experienced cardiac sonographers donned full personal protective equipment as per hospital guidelines (N95 mask, face shield, disposable gown, gloves, hair covers, and shoe covers) and performed focused and detailed TTEs (Figure 1B). These sonographers completed hospital training in procedures for donning and doffing personal protection equipment. Measurements were performed offline, not within the patient's room, to limit time of direct patient exposure. TTE was performed in either the supine or prone position using a focused protocol encompassing all advanced echocardiographic methods. For proned patients, attempts were made to perform TTE in supine position, if possible. Details of this focused ICU TTE protocol for COVID-19 have been published. ${ }^{9}$

All measurements were performed in accordance with American Society of Echocardiography guidelines. ${ }^{10}$ Left ventricular ejection fraction (LVEF) was calculated using the biplane Simpson's method. In patients with adequate
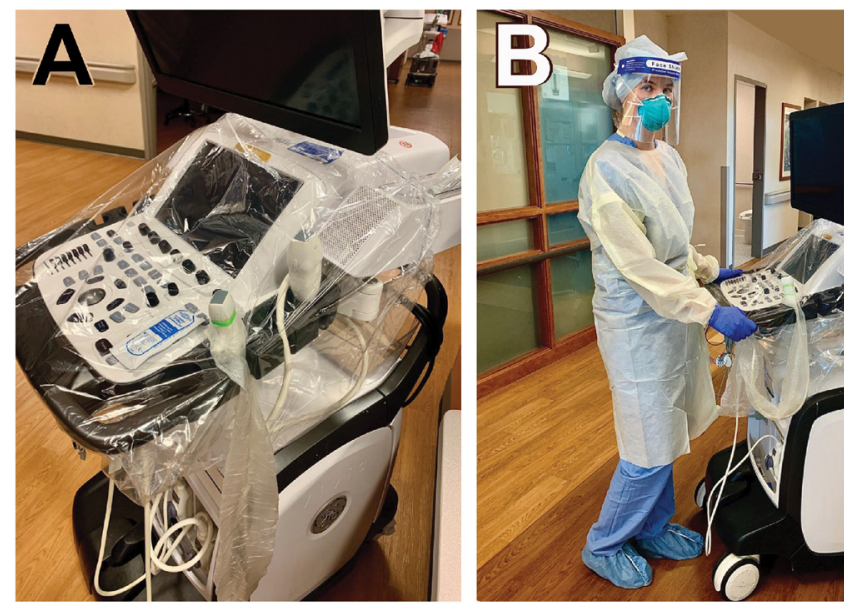

Figure 1. Protection of sonographers. A: Protective equipment for the echocardiography machine includes a keyboard cover, transducer cover, and single-use gel. B: Personal protective equipment for sonographers includes an N95 mask, a face shield, a hair cover, shoe covers, a disposable gown, and gloves.

image quality for speckle-tracking echocardiography, peak left ventricular (LV) global longitudinal strain (GLS) and RV GLS were performed. LV GLS was obtained using standard apical 2-, 3-, and 4-chamber views at frame rates of $>60$ frames per second. RV GLS was performed by tracing the RV endocardial border, then manually averaging 3 segments of RV free wall in an RV-focused apical view at frame rates of $>60$ frames per second. If available, echocardiograms performed pre-COVID illness also were included in the analysis for serial comparison.

The electronic medical record (Epic Systems Corporation) was queried for demographic variables, comorbidities, laboratory results, complications, and details of COVID-related hospitalizations. Based on clinical and echocardiographic data, patients with known cardiomyopathy or coronary disease were grouped into the "known prior cardiac disease" category; all remaining patients were grouped into the "no known prior cardiac disease" category.

\section{Statistical Analysis}

Categorical variables were summarized as counts and proportions. Continuous variables were summarized using mean and standard deviation (SD) or median and interquartile range (IQR) if not assumed to be normally distributed. Proportions were compared across the aforedescribed groups using chi-squared test and means using Student's $t$-test (or their nonparametric equivalents if normality assumption was not fulfilled). 
Statistical significance was defined as a two-sided $P$-value of $<0.05$. All analyses were performed using Stata 15 software (StataCorp LLC).

\section{RESULTS}

Clinical characteristics of 52 patients with COVID-19 admitted to the ICU are listed in Table 1. In this cohort, mean age was 59.9 (SD: 11.6) years, median body mass index was $31.7 \mathrm{~kg} / \mathrm{m}^{2}$ (IQR: 26.4, 36.3), and $31(60 \%)$ patients were men. Our population was racially and ethnically diverse. Common comorbidities included obesity $(\mathrm{n}=27,52 \%)$, hypertension $(\mathrm{n}=36,69 \%)$, and dyslipidemia $(n=35,67 \%)$.

The indication for ICU admission was acute respiratory failure in $96 \%$ of cases $(n=50)$, and $38(73 \%)$ required endotracheal intubation. Hemodynamic instability was the primary indication for ICU admission in the remaining 2 cases (both were noted to have severe LV systolic dysfunction). Patients had evidence of severe inflammation, as noted by the following biomarkers: $70 \%(n=35)$ had elevated D-dimer; $71 \%(n=37)$ had elevated ferritin; and 96\% ( $\mathrm{n}=47)$ had elevated C-reactive protein. The mean Sequential Organ Failure Assessment score on ICU admission was 5.7 (SD: 3.0). Proning strategy was part of routine ICU care and was used in $45(87 \%)$ cases. The most common ICU complication was respiratory failure (48\% developed acute respiratory distress syndrome, the remainder had severe bilateral pneumonia). There were 3 documented cases with thromboembolic complications (deep venous thrombosis, pulmonary embolism, and embolic cerebrovascular incident).

There were 15 (29\%) patients with known prior cardiac disease, some with multiple cardiac conditions - 4 (8\%) had known LV systolic dysfunction, 4 (8\%) had moderate/severe pulmonary hypertension, 2 (4\%) had prior RV dysfunction, 9 (17\%) had obstructive coronary artery disease, $4(8 \%)$ had heart failure with preserved ejection fraction, $2(4 \%)$ had prior heart transplantation, 1 had preexisting severe mitral regurgitation, and 1 had prior moderate aortic stenosis.

All 52 TTEs were performed at the bedside, and 26 $(50 \%)$ patients were intubated at the time of procedure. Fewer than 5 TTEs were done in the prone position, thus, not enough for statistical comparison with TTEs done in the supine position. The majority had TTE performed within 48 hours before and after ICU admission. The mean TTE procedure time was 15.6 (SD: 5.7) minutes, and the mean number of video clips obtained was 45 (SD: 13.2). All echocardiograms were diagnostic of the clinical question, and the predominant indication was evaluation of LV and RV systolic function.
Table 1. Clinical Characteristics of Patients $(\mathrm{N}=52)$

\begin{tabular}{|c|c|}
\hline Variable & Prevalence \\
\hline Age in years & $59.9(11.6)$ \\
\hline 18-39 years & $3(6 \%)$ \\
\hline $40-49$ years & $8(15 \%)$ \\
\hline $50-59$ years & $13(25 \%)$ \\
\hline $60-69$ years & $18(35 \%)$ \\
\hline $70-79$ years & $8(15 \%)$ \\
\hline $80+$ years & $2(4 \%)$ \\
\hline \multicolumn{2}{|l|}{ Race/Ethnicity } \\
\hline Hispanic & $18(35 \%)$ \\
\hline Non-Hispanic White & $15(29 \%)$ \\
\hline Black & $13(25 \%)$ \\
\hline Other & $6(12 \%)$ \\
\hline Male sex & $31(60 \%)$ \\
\hline Tobacco abuse & $22(33 \%)$ \\
\hline Obesity (BMI of >30 kg/m²) & $27(52 \%)$ \\
\hline Atrial fibrillation/Atrial flutter & $6(12 \%)$ \\
\hline Chronic obstructive pulmonary disease & $5(10 \%)$ \\
\hline Coronary artery disease & $9(17 \%)$ \\
\hline Diabetes & $19(37 \%)$ \\
\hline Dyslipidemia & $35(67 \%)$ \\
\hline Heart failure & $11(21 \%)$ \\
\hline Hypertension & $36(69 \%)$ \\
\hline Cancer & $10(19 \%)$ \\
\hline Known prior cardiac disease & $15(29 \%)$ \\
\hline \multicolumn{2}{|l|}{ Laboratory findings } \\
\hline Abnormal troponin (>0.05 ng/mL) & $39(75 \%)$ \\
\hline Abnormal D-dimer (>0.57 mg/L) & $35(70 \%)$ \\
\hline Log-transformed NT-proBNP & $5.79(2.01)$ \\
\hline Abnormal ferritin (>388 ng/mL) & $37(71 \%)$ \\
\hline Abnormal C-reactive protein (>1.0 mg/dL) & $47(96 \%)$ \\
\hline Albumin in $\mathrm{g} / \mathrm{dL}$ & $2.96(0.44)$ \\
\hline \multicolumn{2}{|l|}{ Prehospitalization medications } \\
\hline ARB/ACEi inhibitor & $18(35 \%)$ \\
\hline Diuretic & $18(35 \%)$ \\
\hline Calcium channel blocker & $13(25 \%)$ \\
\hline Beta blocker & $14(27 \%)$ \\
\hline Statin & $29(56 \%)$ \\
\hline \multicolumn{2}{|l|}{ COVID-19 treatments } \\
\hline Tocilizumab & $26(50 \%)$ \\
\hline Convalescent plasma & $26(50 \%)$ \\
\hline Remdesivir & $9(17 \%)$ \\
\hline Hydroxychloroquine & $33(63 \%)$ \\
\hline Azithromycin & $34(65 \%)$ \\
\hline Steroids & $33(63 \%)$ \\
\hline
\end{tabular}

Data presented as $n(\%)$ or mean (SD). Percentages based off total number of patients with that variable measured.

$A R B / A C E i$, angiotensin receptor blocker/angiotensinconverting enzyme inhibitor; BMI, body mass index; NT-proBNP, N-terminal pro-B-type natriuretic peptide. 
Cardiac complications occurred in $56 \%$ of those admitted to the ICU. Acute ventricular dysfunction (isolated left, isolated right, or biventricular dysfunction) developed in $40 \%$ of patients (3 isolated new or worsening LV systolic dysfunction, 10 isolated new or worsening RV systolic dysfunction assessed visually, and 8 with combined LV and RV systolic dysfunction). New-onset atrial fibrillation developed in 6 patients. In 1 patient with known severe LV systolic dysfunction, ventricular tachycardia developed, necessitating medical intervention. One patient's ICU course was complicated by non-ST-elevation myocardial infarction. No hemodynamically significant valvular abnormalities were noted in these studies, and no cases of endocarditis were seen.

On echocardiogram after ICU admission (Table 2), LVEF was relatively preserved (median: 57\%; IQR:
$50 \%, 64 \%)$. Yet, 1 in 5 patients $(n=11)$ had new or worsening LV systolic dysfunction (measured by an LVEF decrease of $>10 \%$ ). There were 9 patients with new abnormal LVEF (ie, $<50 \%$ ); of the 4 patients with known LV dysfunction, 2 had acute worsening of LVEF by $>10 \%$. All were globally hypokinetic. Subclinical LV dysfunction was prevalent, as both LV GLS and myocardial global work index were abnormal. Patients with known prior cardiac disease ( $40 \%$ vs $14 \%$ with no known prior cardiac disease; $\mathrm{P}=0.03$ ) were more likely to have new or worsening LV systolic dysfunction and more abnormal myocardial mechanics (LV GLS, global myocardial work index) (Table 3).

The most prevalent abnormality on TTE was abnormal $\mathrm{RV}$ size and function. RV enlargement was present in $20(38 \%)$ patients. New or worsening RV dysfunction

Table 2. Echocardiographic Findings in COVID-19 Patients in the Intensive Care Unit ( $N=52)$

\begin{tabular}{|c|c|c|}
\hline Parameter & $\begin{array}{c}\text { Total cases with } \\
\text { parameter measured }\end{array}$ & $\begin{array}{c}\text { Mean (IQR) or } \\
\text { parameter-positive } \mathrm{n}(\%)\end{array}$ \\
\hline \multicolumn{3}{|l|}{ LV echocardiographic parameters } \\
\hline LVEF & 47 & $0.57(0.5,0.64)$ \\
\hline Body surface area, $\mathrm{m}^{2}$ & 52 & $2.08(1.82,2.27)$ \\
\hline End-diastolic volume, $\mathrm{mL}$ & 38 & $97.04(78.07,122.5)$ \\
\hline End-systolic volume, mL & 38 & $44.45(31.14,53.49)$ \\
\hline E' septal, $\mathrm{cm} / \mathrm{s}$ & 20 & $7.29(5.70,8.55)$ \\
\hline LV GLS, \% & 30 & $-16.00(-17,-14.3)$ \\
\hline Left atrial volume index & 10 & $35.87(23.76,49.40)$ \\
\hline Cardiac index & 17 & $4.53(4.01,6.24)$ \\
\hline LV filling pressure, E/e' & 20 & $10.49(7.22,14.745)$ \\
\hline Global work index, $\mathrm{mmHg} \%$ & 36 & $1515(1283,1657.5)$ \\
\hline Global work efficiency, \% & 36 & $93(89,94.5)$ \\
\hline New or worsening LV dysfunction & 52 & $11(21 \%)$ \\
\hline \multicolumn{3}{|l|}{ RV echocardiographic parameters } \\
\hline Diastolic area, $\mathrm{cm}^{2}$ & 9 & $21.89(15.5,23.9)$ \\
\hline Systolic area, $\mathrm{cm}^{2}$ & 9 & $16.37(9.35,17.26)$ \\
\hline Fractional area change & 10 & $0.32(0.18,0.40)$ \\
\hline TAPSE, mm & 13 & $18.12(17.39,22.7)$ \\
\hline $\mathrm{S}^{\prime}$ velocity, $\mathrm{cm} / \mathrm{s}$ & 44 & $0.13(0.102,0.17)$ \\
\hline RV GLS, \% & 36 & $-19.50(-23,-15.2)$ \\
\hline Abnormal RV GLS & 36 & $28(78 \%)$ \\
\hline Visual assessment of RV dysfunction & 52 & $18(35 \%)$ \\
\hline RV dysfunction (all parameters) ${ }^{\mathrm{a}}$ & 52 & $34(65 \%)$ \\
\hline Pulmonary hypertension ${ }^{\mathrm{b}}$ & 36 & $10(19 \%)$ \\
\hline RV cavity enlargement & 52 & $20(38 \%)$ \\
\hline
\end{tabular}

${ }^{a}$ Any abnormal value of RV GLS, TAPSE, fractional area change, or S' velocity.

${ }^{b}$ Pulmonary artery systolic pressure of $>40 \mathrm{mmHg}$.

GLS, global longitudinal strain; IQR, interquartile range; LV, left ventricular; LVEF, left ventricular ejection fraction; $R V$, right ventricular; TAPSE, tricuspid annular plane systolic excursion. 
Table 3. Echocardiographic Findings in ICU Patients With COVID-19 by Known Status of Prior Cardiac Disease

\begin{tabular}{|c|c|c|c|}
\hline & $\begin{array}{c}\text { No known prior } \\
\text { cardiac disease, } n=37\end{array}$ & $\begin{array}{c}\text { Known prior cardiac } \\
\text { disease, } n=15\end{array}$ & $P$ \\
\hline \multicolumn{4}{|l|}{ LV echocardiographic parameters } \\
\hline LVEF & $0.58(0.55,0.63)$ & $0.51(0.45,0.65)$ & 0.10 \\
\hline Body surface area, $\mathrm{m}^{2}$ & $2.00(1.80,2.17)$ & $2.24(1.86,2.39)$ & 0.05 \\
\hline End-diastolic volume, $\mathrm{mL}$ & $95.39(77.99,114.05)$ & $117.45(85.54,143.4)$ & 0.22 \\
\hline End-systolic volume, mL & $41.30(29.83,50.78)$ & $56.02(45.56,69.2)$ & 0.04 \\
\hline E' septal, cm/s & $7.55(5.77,8.55)$ & $6.60(4.65,9.28)$ & 0.59 \\
\hline LV GLS, \% & $-16.50(-18.50,-15)$ & $-14.25(-15.25,-9.05)$ & $<0.01$ \\
\hline Left atrial volume index & $23.84(23.21,43.73)$ & $53.76(42.31,57.19)$ & 0.05 \\
\hline Cardiac index & $46.23(40.15,62.41)$ & $45.32(38.37,62.47)$ & 0.99 \\
\hline LV filling pressure, E/e' & $9.78(6.47,11.85)$ & $14.20(8.75,20.22)$ & 0.09 \\
\hline Global work index, mmHg\% & $1605(1351,1697)$ & $1276(756,1469)$ & 0.02 \\
\hline Global work efficiency, \% & $94(89,95)$ & $92(90,93)$ & 0.37 \\
\hline New or worsening LV dysfunction & $5(14 \%)$ & $6(40 \%)$ & 0.03 \\
\hline \multicolumn{4}{|l|}{ RV echocardiographic parameters } \\
\hline Diastolic area, $\mathrm{cm}^{2}$ & $19.70(15.5,23.92)$ & $23.77(14.33,23.9)$ & 0.99 \\
\hline Systolic area, $\mathrm{cm}^{2}$ & $12.86(9.25,16.81)$ & $19.51(12.09,20.68)$ & 0.12 \\
\hline TAPSE, mm & $17.695(15.825,22.37)$ & $22(18.12,22.7)$ & 0.21 \\
\hline $\mathrm{S}^{\prime}$ velocity, $\mathrm{cm} / \mathrm{s}$ & $0.13(0.10,0.17)$ & $0.13(0.09,0.15)$ & 0.43 \\
\hline RV GLS, \% & $-19(-23,-16)$ & $-20(-22.50,-14)$ & 0.69 \\
\hline Visual assessment of RV dysfunction & $11(30 \%)$ & $7(47 \%)$ & 0.25 \\
\hline RV dysfunction (all parameters) & $23(62 \%)$ & $11(73 \%)$ & 0.44 \\
\hline Pulmonary hypertension ${ }^{a}$ & $4(11 \%)$ & $6(40 \%)$ & 0.02 \\
\hline RV cavity enlargement & $12(32 \%)$ & $8(53 \%)$ & 0.16 \\
\hline
\end{tabular}

Data presented as median (interquartile range) or $n(\%)$.

${ }^{a}$ Pulmonary artery systolic pressure of $>40 \mathrm{mmHg}$.

GLS, global longitudinal strain; ICU, intensive care unit; LV, left ventricular; LVEF, left ventricular ejection fraction; $R V$, right ventricular; TAPSE, tricuspid annular plane systolic excursion.

(assessed qualitatively) was noted in 18 (35\%) patients; of these, $8(15 \%)$ had both LV and RV dysfunction. When combined with echocardiography-derived parameters (RV S' velocity, RV tricuspid annular plane systolic excursion, RV GLS, RV fractional area change), 34 (65\%) cases had some measure of abnormal RV function. Whether in prone or supine position, additional RV echocardiographic parameters were obtainable in the majority of patients (Figure 2). RV GLS was abnormal in $78 \%$ of cases in which RV GLS could be obtained ( $n=36$; median: -19.50; IQR: -23, -15.2). In patients with known prior cardiac disease, RV function was worse and pulmonary artery systolic pressure was higher (Table 3 ).

Mean ICU stay was 15.5 (SD: 11.8) days, and mean hospital length of stay was 21.7 (SD: 14.6) days. Follow-up was available on all patients: overall hospital mortality was $27 \%(n=14), 42 \%(n=22)$ of patients were discharged to their home, and $31 \%(n=16)$ of patients were discharged to a long-term acute care facility (of whom 7 had a tracheostomy). The 2 patients who received venovenous extracorporeal membrane oxygenation died from respiratory failure. Prolonged ventilator time was associated with worse clinical outcomes. Compared to those discharged home (Table 4), patients who died or were discharged to long-term care were more likely to be older (mean age: $54.9 \pm 8.8$ years vs $63.6 \pm 12.2$ years; $\mathrm{P}<0.01$ ), have clinical history of atrial fibrillation/atrial flutter $(0 \%$ vs $20 \% ; \mathrm{P}=0.03)$, have hypertension $(55 \%$ vs $80 \% ; \mathrm{P}=0.05)$, and have known prior cardiac disease ( $14 \%$ vs $40 \%, \mathrm{P}=0.04)$.

LV function, as assessed by echocardiography, was not associated with morbidity and mortality in our small study. On the RV side, compared to those discharged home, patients who died or were discharged to long-term care were more likely to have pulmonary hypertension $(5 \%$ vs $30 \% ; \mathrm{P}=0.02)$ and $\mathrm{RV}$ cavity enlargement $(23 \%$ 


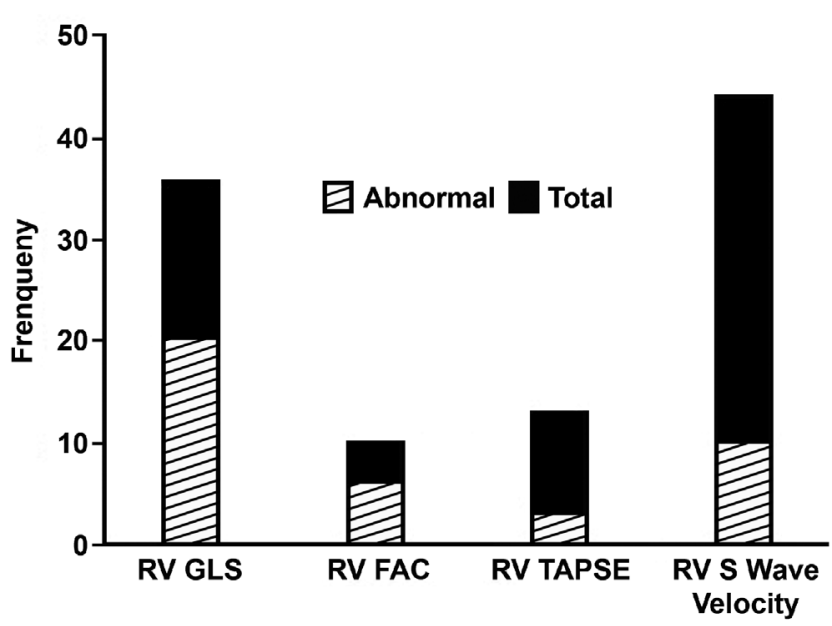

Figure 2. Ability to perform quantitative echocardiographic measurements of right ventricular $(R V)$ function. $S^{\prime}$ velocity was the most readily obtained measurement of $R V$ function. $R V$ fractional area change (FAC) was the least easily obtained. Abnormal global longitudinal strain (GLS) was the most frequent abnormality of RV dysfunction. TAPSE, tricuspid annular plane systolic excursion.

vs $50 \% ; \mathrm{P}=0.05)$. Although not statistically significant, given low numbers, median RV GLS was more abnormal in patients who died or were discharged to long-term care. Abnormal RV GLS was present in 28 of the 36 patients with GLS values (of those 28, 9 died or were discharged to long-term care). More patients with new or worsening RV dysfunction also were in the group of patients who died or were discharged to long-term care. Finally, in the lowest tertile of RV GLS (ie, $\geq-15 \%$ ), there was a trend toward longer hospital stay and longer ICU stay (median hospital stay: 20 [IQR: 13.75, 37.5] days vs 17.5 [IQR: 10,28] days; median ICU stay: 14.5 [IQR: 9, 26.25] days vs 9 [IQR: 5.25, 20.75] days).

\section{DISCUSSION}

Patients requiring intensive care form a subset of the COVID-19 pandemic that has a severe and often multiorgan system response to the virus. In our ICUadmitted population, median ICU and total hospital stays were long ( $>2$ weeks and $>3$ weeks, respectively), with severe respiratory failure and prolonged ventilation. ICU hospitalizations were not only associated with significant mortality (27\%) but also significant morbidity, as $31 \%$ of patients could not return home after hospital discharge.

Echocardiography plays a crucial role in identifying cardiovascular complications in patients admitted to the ICU with COVID-19. Use of advanced echocardiographic technologies identified clinical and subclinical abnormalities of heart function, changing clinical management and guiding ICU strategy. These imaging tests were performed by a core group of experienced cardiac sonographers on state-of-the-art echocardiography machines using a focused protocol, rather than pointof-care ultrasound or portable ultrasound machines. As published previously, the mean scan time to complete this protocol was 15 (SD: 5.6) minutes, ${ }^{9}$ and none of the core group contracted the virus. Cardiac sonographers are at significant risk of COVID-19 exposure due to their proximity to the patient. Yet, if protected properly with appropriate personal protective equipment, a focused test can be performed quickly and with the latest ultrasound techniques, providing guidance and risk stratification to ICU teams dealing with the COVID-19 pandemic. ${ }^{9}$

Although the primary etiology of morbidity/ mortality is from respiratory failure, cardiac disease is not a bystander in critically ill COVID-19 patients. Cardiovascular complications were very common in our study and included isolated LV dysfunction, isolated RV dysfunction, biventricular dysfunction, and atrial and ventricular arrythmias. Similar findings have been reported from other centers. ${ }^{11,12}$ Multiple mechanisms are implicated in cardiac complications: 1) oxygen-demand mismatch leading to coronary ischemia, 2) inflammatory cascade leading to increased ischemic or embolic events, and 3) direct myocardial injury caused by the virus entering myocytes. ${ }^{13}$ Elevated N-terminal pro-B-type natriuretic peptide, a marker of heart failure, also was associated with worse clinical outcomes in our patients. The types of complications we observed were likely a result of multiple pathways leading to cardiac injury.

Known prior cardiac disease was associated with increased morbidity and mortality in ICU-admitted patients with COVID-19, similar to experience in China ${ }^{5}$ and Italy. ${ }^{6}$ Known prior cardiac disease, in the Italian study, was associated with more than double the mortality in all hospitalizations. In our study, we focused on ICU patients exclusively and demonstrated that known prior cardiac disease was associated with higher mortality and also higher morbidity (ie, more patients with known cardiac disease required long-term acute care, even if they survived hospitalization). This is particularly true of left-sided disease, as LV dysfunction at baseline was associated with acute worsening of LVEF during ICU hospitalization.

The most common cardiac complication in our study was RV enlargement and dysfunction. In contrast to leftsided disease, this complication was independent of a history of known prior cardiac disease. This is consistent with a prior study, in which RV dilation was the only echocardiographic finding to prognosticate mortality in 
Table 4. Comparison of ICU Patients Discharged Home With Those Having Significant Morbidity and Mortality (Death or Discharged to LTAC)

\begin{tabular}{|c|c|c|c|}
\hline Variable & $\begin{array}{l}\text { Alive, discharged home } \\
n=22\end{array}$ & $\begin{array}{c}\text { Dead or in LTAC } \\
n=30\end{array}$ & $P$ \\
\hline Age in years & $54.9(8.8)$ & $63.6(12.2)$ & $<0.01$ \\
\hline \multicolumn{4}{|l|}{ Race/Ethnicity } \\
\hline Hispanic & $9(41 \%)$ & $9(30 \%)$ & \\
\hline Non-Hispanic White & $5(23 \%)$ & $10(33 \%)$ & \\
\hline Black & $4(18 \%)$ & $9(30 \%)$ & \\
\hline Other & $4(18 \%)$ & $2(7 \%)$ & 0.38 \\
\hline Male sex & $11(50 \%)$ & $20(67 \%)$ & 0.23 \\
\hline Tobacco abuse & $5(24 \%)$ & $17(56 \%)$ & 0.11 \\
\hline Obesity (BMI of >30 kg/m²) & $14(67 \%)$ & $13(52 \%)$ & 0.31 \\
\hline Atrial fibrillation/Atrial flutter & $0(0 \%)$ & $6(20 \%)$ & 0.03 \\
\hline COPD & $1(5 \%)$ & $4(13 \%)$ & 0.29 \\
\hline Coronary artery disease & $2(9 \%)$ & $7(23 \%)$ & 0.18 \\
\hline Diabetes & $8(36 \%)$ & $11(37 \%)$ & 0.98 \\
\hline Dyslipidemia & $12(55 \%)$ & $23(77 \%)$ & 0.09 \\
\hline Heart failure & $2(9 \%)$ & $9(30 \%)$ & 0.07 \\
\hline Hypertension & $12(55 \%)$ & $24(80 \%)$ & 0.05 \\
\hline Cancer & $2(9 \%)$ & $8(27 \%)$ & 0.11 \\
\hline Known prior cardiac disease & $3(14 \%)$ & $12(40 \%)$ & 0.04 \\
\hline Log-transformed NT-proBNP & $91(44.8,360.8)$ & $673.5(93.3,2774.3)$ & 0.03 \\
\hline Time on ventilator in days & $7(5.5,9)$ & $15(6.5,27)$ & 0.03 \\
\hline \multicolumn{4}{|l|}{ LV echocardiographic parameters } \\
\hline LVEF & $0.56(0.52,0.6)$ & $0.59(0.47,0.65)$ & 0.48 \\
\hline Body surface area, $\mathrm{m}^{2}$ & $2.02(1.80,2.22)$ & $2.08(1.86,2.27)$ & 0.59 \\
\hline End-diastolic volume, $\mathrm{mL}$ & $89.8(74.06,122.5)$ & $109.4(87.68,128.7)$ & 0.18 \\
\hline End-systolic volume, mL & $41.27(27.79,53.49)$ & $48.9(37.11,58.83)$ & 0.26 \\
\hline E' septal, $\mathrm{cm} / \mathrm{s}$ & $7.58(6.20,8.39)$ & $6.60(5.32,10.12)$ & 0.94 \\
\hline LV GLS, \% & $-16(-17,-14.50)$ & $-15(-17,-14)$ & 0.36 \\
\hline Left atrial volume index & $29.43(23.84,53.76)$ & $42.31(23.21,49.40)$ & 0.73 \\
\hline Cardiac index & $47.62(37.62,57.63)$ & $45.32(42.71,62.47)$ & 0.56 \\
\hline LV filling pressure, E/e' & $10.77(8.72,15.52)$ & $10.19(6.55,14.58)$ & 0.59 \\
\hline Global work index, mmHg\% & $1623.5(1341.5,1769)$ & $1433.5(1267.5,1605.5)$ & 0.18 \\
\hline Global work efficiency, \% & $94(91.5,95.5)$ & $90.5(88,94)$ & 0.07 \\
\hline New or worsening LV dysfunction & $5(23 \%)$ & $6(20 \%)$ & 0.81 \\
\hline \multicolumn{4}{|l|}{ RV echocardiographic parameters } \\
\hline Diastolic area, $\mathrm{cm}^{2}$ & $17.5(15.5,23.9)$ & $22.83(14.33,23.92)$ & 0.80 \\
\hline Systolic area, $\mathrm{cm}^{2}$ & $9.35(9.25,20.68)$ & $16.59(12.09,17.26)$ & 0.80 \\
\hline Fractional area change & $0.40(0.13,0.47)$ & $0.32(0.18,0.37)$ & 0.57 \\
\hline TAPSE, mm & $21(18.12,25.43)$ & $18(16.70,22.35)$ & 0.30 \\
\hline S' velocity, $\mathrm{cm} / \mathrm{s}$ & $0.12(0.10,0.15)$ & $0.14(0.10,0.17)$ & 0.49 \\
\hline RV GLS, \% & $-20(-22,-15)$ & $19(-23,-15.90)$ & 0.97 \\
\hline RV dysfunction (all parameters) ${ }^{a}$ & $13(59 \%)$ & $21(70 \%)$ & 0.41 \\
\hline Pulmonary hypertension ${ }^{b}$ & $1(5 \%)$ & $9(30 \%)$ & 0.02 \\
\hline RV cavity enlargement & $5(23 \%)$ & $15(50 \%)$ & 0.05 \\
\hline Visual assessment of RV dysfunction & $7(32 \%)$ & $11(37 \%)$ & 0.72 \\
\hline
\end{tabular}

Data presented as $n$ (\%), mean (standard deviation), or median (interquartile range).

${ }^{a}$ Any abnormal value of RV GLS, TAPSE, fractional area change, or S' velocity.

${ }^{b}$ Pulmonary artery systolic pressure of $\geq 40 \mathrm{mmHg}$.

BMI, body mass index; COPD, chronic obstructive pulmonary disease; GLS, global longitudinal strain; ICU, intensive care unit; LTAC, long-term acute care facility; LV, left ventricular; LVEF, left ventricular ejection fraction; NT-proBNP, N-terminal pro-B-type natriuretic peptide; $R V$, right ventricular; TAPSE, tricuspid annular plane systolic excursion. 
hospitalized patients with COVID-19 (odds ratio: 4.5, 95\% CI: 1.5-13.7). ${ }^{8}$ In our study, RV enlargement was associated with both mortality and significant morbidity in those who were critically ill. In the Wuhan, China, study, bedside echocardiography was performed on patients with COVID-19, up to a week into admission, and detailed echocardiographic measurements including myocardial mechanics were performed. ${ }^{7}$ Of note and in contrast to our study, patients with known prior cardiac disease were excluded. A threshold RV GLS of $-23 \%$ was observed as a cutoff value, and values greater than $-23 \%$ were associated with higher mortality. ${ }^{7}$ We used this same value in our study and noted that abnormal RV GLS was present in 28 of the 36 patients with recorded GLS values. In a New York series, portable ultrasound was used and RV dilation was defined semiquantitatively. ${ }^{8}$ As in the Wuhan study, we chose not to use portable ultrasound. Instead, we used latest-generation echocardiography machines; therefore, multiple abnormalities of RV function could be identified, including cases that would be missed if only visual assessment of RV function was performed. If only point-of-care or limited portable ultrasound machines were used, RV dysfunction would have been detected in just 18 patients. Visual assessment of RV function is usually noted by the echocardiographer observing reduced RV radial function, seen in apical and subcostal views; however, using state-of-the-art echocardiographic technology, RV dysfunction can be assessed with multiple well-validated assessments of $\mathrm{RV}$ function. We therefore noted RV dysfunction in the majority (65\%) of ICU patients with COVID-19.

$\mathrm{RV}$ function has been implicated in prior studies of acute respiratory distress syndrome as a contributor to morbidity, ${ }^{14}$ and a similar mechanism of hypoxic vasoconstriction to that seen in acute respiratory distress syndrome may also be seen in COVID-19. SARSCoV-2 may directly affect RV myocytes, leading to RV myocarditis. Finally, COVID-19 is associated with thromboembolic complications, and microscopic pulmonary emboli may contribute to RV dysfunction. Szekely et al noted that worsening RV dysfunction was associated with clinical deterioration in hospitalized COVID-19 patients. $^{15}$ Further research is needed to evaluate mechanisms of RV dilation and dysfunction as well as treatment strategies.

This identification of pulmonary hypertension and RV dysfunction by echocardiography was crucial in guiding ICU practice. When RV dysfunction was identified in our ICU, single or multiple strategies were employed to minimize mortality from RV dysfunction - afterload reduction with nitric oxide, dobutamine for ionotropic support, and even more conservative fluid management. Epoprostenol was not used to minimize virus aerosolization that could occur from breaking the ventilator circuit. Positive end-expiratory pressure ventilation also was limited. Although beyond the scope of this study, future studies could evaluate the benefit of anticoagulation on RV function in hospitalized patients with COVID-19.

\section{Limitations}

This is a single-center study evaluating echocardiographic variables in patients with COVID-19 illness who required ICU admission. This study is limited to only those patients with COVID-19 in whom the primary provider clinically assessed a need for an echocardiogram; however, as part of routine clinical care, most patients admitted to the ICU did receive limited TTE. This study is also limited by the fact that patients without a previous echocardiogram available were categorized as no known prior cardiac disease but may in fact have had prior cardiac disease that was not diagnosed.

\section{CONCLUSIONS}

ICU admission for COVID-19 is associated with significant morbidity and mortality in an ethnically diverse population. Echocardiography has an additive role in identifying complications and highlighting patients who require further care. The most common echocardiographic abnormality is right ventricular dysfunction, which can be assessed more accurately using state-of-the-art echocardiography. Known prior cardiac disease, relatively older age, atrial fibrillation/atrial flutter, RV enlargement, and pulmonary hypertension are associated with worse outcomes.

\section{Patient-Friendly Recap}

- Patients diagnosed with COVID-19 who are admitted to the intensive care unit (ICU) are at significant risk of dying or requiring long-term acute care.

- Cardiovascular complications are common in these patients and often identified using echocardiographic imaging.

- In addition to having a history of prior cardiac disease, the presence of pulmonary hypertension, atrial fibrillation/flutter, or enlarged right ventricle as measured by echocardiography increases the risk of poor outcomes for those hospitalized in the ICU with COVID-19.

\section{Acknowledgments}

The authors gratefully acknowledge the cardiac sonographers at Aurora St. Luke's Medical Center for their tireless dedication in advancing cardiac care for patients hospitalized with COVID-19. 
The authors also acknowledge the following support staff from Aurora Cardiovascular and Thoracic Services: Jennifer Pfaff and Susan Nord for editorial preparation of the manuscript and Brian Miller and Brian Schurrer for assistance with the figures.

\section{Author Contributions}

Study design: Jain, Salinas, Kroboth, Perez Moreno, Khandheria. Data acquisition or analysis: Jain, Salinas, Kroboth, Kaminski, Roemer, Perez Moreno. Manuscript drafting: Jain, Kroboth, Kaminski, Roemer. Critical revision: Jain, Salinas, Kroboth, Perez Moreno, Khandheria.

\section{Conflicts of Interest}

None.

\section{References}

1. Clerkin KJ, Fried JA, Raikhelkar J, et al. COVID-19 and cardiovascular disease. Circulation. 2020;141:1648-55. CrossRef

2. Inciardi RM, Lupi L, Zaccone $\mathrm{G}$, et al. Cardiac involvement in a patient with coronavirus disease 2019 (COVID-19). JAMA Cardiol. 2020;5:819-24. CrossRef

3. Deng Q, Hu B, Zhang Y, et al. Suspected myocardial injury in patients with COVID-19: evidence from front-line clinical observation in Wuhan, China. Int J Cardiol. 2020;311:116-21. CrossRef

4. Zeng JH, Liu YX, Yuan J, et al. First case of COVID-19 complicated with fulminant myocarditis: a case report and insights. Infection. 2020;48:773-7. CrossRef

5. Shi S, Qin M, Shen B, et al. Association of cardiac injury with mortality in hospitalized patients with COVID-19 in Wuhan, China. JAMA Cardiol. 2020;5:802-10. CrossRef

6. Inciardi RM, Adamo M, Lupi L, et al. Characteristics and outcomes of patients hospitalized for COVID-19 and cardiac disease in Northern Italy. Eur Heart J. 2020;41:1821-9. CrossRef
7. Li Y, Li H, Zhu S, et al. Prognostic value of right ventricular longitudinal strain in patients with COVID-19. JACC Cardiovasc Imaging. 2020;13:2287-99. CrossRef

8. Argulian E, Sud K, Vogel B, et al. Right ventricular dilation in hospitalized patients with COVID-19 infection. JACC Cardiovasc Imaging. 2020;13:2459-61. CrossRef

9. Kaminski A, Payne A, Roemer S, Ignatowski D, Khandheria BK. Answering to the call of critically ill patients: limiting sonographer exposure to COVID-19 with focused protocols. J Am Soc Echocardiogr. 2020;33:902-3. CrossRef

10. Lang RM, Badano LP, Mor-Avi V, et al. Recommendations for cardiac chamber quantification by echocardiography in adults: an update from the American Society of Echocardiography and the European Association of Cardiovascular Imaging. $J \mathrm{Am}$ Soc Echocardiogr. 2015;28:1-39.e14. CrossRef

11. Mahmoud-Elsayed HM, Moody WE, Bradlow WM, et al. Echocardiographic findings in patients with COVID-19 pneumonia. Can J Cardiol. 2020;36:1203-7. CrossRef

12. Jain SS, Liu Q, Raikhelkar J, et al. Indications for and findings on transthoracic echocardiography in COVID-19. J Am Soc Echocardiogr. 2020;33:1278-84. CrossRef

13. Rudski L, Januzzi JL, Rigolin VH, et al. Multimodality imaging in evaluation of cardiovascular complications in patients with COVID-19: JACC Scientific Expert Panel. J Am Coll Cardiol. 2020;76:1345-57. CrossRef

14. Lazzeri C, Bonizzoli M, Cianchi G, Batacchi S, Chiostri M, Peris A. Severity of acute respiratory distress syndrome and echocardiographic findings in clinical practice - an echocardiographic pilot study. Heart Lung. 2020;49:622-5. CrossRef

15. Szekely Y, Lichter Y, Taieb P, et al. Spectrum of cardiac manifestations in COVID-19: a systematic echocardiographic study. Circulation. 2020;142:342-53. CrossRef

(C) 2021 Advocate Aurora Health, Inc. 